ANUARIO DE ESTUdIOS MEDIEVALES

50/1, enero-junio de 2020, pp. 267-292

ISSN 0066-5061

https://doi.org/10.3989/aem.2020.50.1.10

\title{
IL FANTASMA DELLA CANTIGA DE SANTA MARÍA 72: MODELLI CULTURALI E FONTI LETTERARIE
}

\author{
THE GHOST OF CANTIGA DE SANTA MARÍA 72: \\ CULTURAL MODELS AND LITERARY SOURCES
}

\author{
MANUEL NEGRI \\ Universidade de Santiago de Compostela \\ University of Oxford (Academic Visitor) \\ https://orcid.org/0000-0003-3532-9810
}

\begin{abstract}
Riassunto: Tra le Cantigas de Santa María, collezione di miracoli mariani compilata da Alfonso X e i suoi collaboratori nella seconda metà del sec. XIII, la cantiga 72 presenta uno strano caso di manifestazione soprannaturale. In essa si narra l'apparizione di un fantasma con il fine di indicare ad un uomo il luogo esatto dove giace il cadavere del figlio, giustiziato da Dio per un peccato di blasfemia. La critica non ha saputo fornire una risposta chiara sulla natura di questo spettro e soprattutto sulle ragioni della sua manifestazione, nonché la giustificazione del suo ruolo nella linea narrativa. Partendo da contributi apparsi in precedenza, questo lavoro cercherà di offrire qualche risposta più articolata sulla questione. Lo si farà a partire da un'analisi della cantiga 72 che tenga conto anche di quelle fonti che l'avrebbero potuta ispirare, così come di quelle credenze religiose che potrebbero sostenere l'intera tradizione miracolistica.
\end{abstract}

Parole chiave: Cantigas de Santa María; Alfonso X; cantiga 72; fantasma; Speculum Historiale.

Abstract: A curious case of supernatural manifestation is presented in cantiga 72, one of the Cantigas de Santa María -a collection of Marian miracles compiled by Alfonso X and his entourage in the second half of the $13^{\text {th }}$ century. Cantiga 72 depicts what appears to be the apparition of a ghost in front of a man to inform him about the place where his son's body lies, his son having been sentenced to death by God after being judged guilty of blasphemy. Scholarship has not been able to give a clear answer to the nature of this ghost, the reasons behind its manifestation and the justification of its role in the narrative. The aim of this paper is to offer some answers to these questions through a close analysis of cantiga 72 , which takes into account those sources and traditions that could have contributed to the inclusion of this supernatural manifestation in this Galician-Portuguese miracle.

Keywords: Cantigas de Santa María; Alfonso X; cantiga 72; ghost; Speculum Historiale.

\section{SOMMARIO}

1. Apparizioni e la Cantiga de Santa María 72.- 2. Un'apparizione non sempre trasparente: letture precedenti.- 3. Una rivelazione nella tradizione.- 3.1. La cantiga 72 e la tradizione dello Speculum Historiale.- 3.2. Il modello 'petrino' sullo sfondo.4. Osservazioni e conclusioni.- 5. Bibliografia citata.

Citation / Cómo citar este artículo: Negri, Manuel (2020), Il fantasma della Cantiga de Santa Maria 72: modelli culturali e fonti letterarie, "Anuario de Estudios Medievales" 50/1, pp. 267-292. https://doi.org/10.3989/aem.2020.50.1.10

Copyright: (C) 2020 CSIC. This is an open-access article distributed under the terms of the Creative Commons Attribution 4.0 International (CC BY 4.0) License. 


\section{ApPARIZIONI E LA CANTIGA DE SANTA MARÍA $72^{1}$}

Le Cantigas de Santa María trasmettono un gran numero di prodigi in cui si narra la discesa o la manifestazione nel mondo terreno -in modalità sempre differenti- della Vergine, di santi o addirittura di demoni, quest'ultimi pronti a contendersi con le forze celesti le anime di fedeli inizialmente inadempienti nei confronti della Vergine Maria.

Tra le più comuni, appartenenti a tradizioni altomedievali fissatesi precocemente nel panorama miracolistico in collezioni di prodigi mariani largamente diffuse, si ricordino, ad esempio, quelle aventi per protagonisti quei santi particolarmente devoti alla Madre di Dio che non esitano a manifestarsi per aiutarla nelle sue attività di redenzione del genere umano. Le CSM mostrano una casistica alquanto nutrita in merito a questi santi "protagonisti", dando notizia, ad esempio, in un nuovo contesto narrativo dedicato alla Vergine, delle gesta post mortem di famosi martiri come San Mercurio (apparso, armato di tutto punto, per uccidere l'apostata Giovanni nella CSM 15) ${ }^{2}$, o della manifestazione in somnis dello stesso San Fernando, padre di Alfonso X, per dare a quest'ultimo alcune istruzioni ${ }^{3}$.

Accanto a queste si ricordino le numerose descrizioni di apparizioni angeliche che sovente accompagnano il corteo celeste di Maria quando questa si manifesta all'interno di una chiesa (è il caso del miracolo avente per protagonista San Bonus nella CSM 66). Il Diavolo è protagonista anch'esso di un gran numero di apparizioni aventi la finalità di far cadere nel peccato chi vacilla nella fede o di contendersi la sua anima con un altro santo, come accade, ad esempio, nel famoso caso del pellegrino suicida della CSM $26^{4}$.

Ma oltre alle tante apparizioni di questo tipo che riguardano -riprendendo la definizione di Jean-Claude Schmitt- "morti di élite"5 le cui manifestazioni erano ritenute del tutto normali e perfettamente inquadrabili nella logica

\footnotetext{
${ }^{1}$ Abbreviazioni utilizzate $\mathrm{c} .=$ carta $; \mathrm{cc} .=$ carte $;$ coll.$=$ collezione $; \mathrm{CSM}=$ Cantiga de Santa María; $C S M=$ Cantigas de Santa María $; \mathrm{ms} .=$ manoscritto; $\mathrm{mss} .=$ manoscritti $; \mathrm{v} .=$ verso; vv. = versi. Questo articolo sviluppa le conclusioni parziali esposte in un congresso organizzato a Santiago de Compostela nel mese di aprile del 2018. Sono grato alla prof. Elvira Fidalgo Francisco per aver dimostrato interesse nella lettura della prima versione di questo testo e per i suoi preziosi consigli.

${ }^{2}$ Per questa figura all'interno delle CSM, si rimanda al classico Montoya 1998-1999.

${ }^{3}$ Su questa casistica, è conveniente rimandare a Negri 2017, dove vengono analizzate le modalità di intervento e le varie tipologie di questi "santi protagonisti", soprattutto in relazione alle fonti delle CSM.

${ }^{4}$ Sul diavolo nelle CSM, si veda Fidalgo 2013. Sul caso del pellegrino, si rimanda ai contributi di Brea 2002, 2005.

${ }^{5}$ Schmitt 1982; 1988a, pp. 183-184.
} 
della salvezza fin dai primi tempi del cristianesimo ${ }^{6}$, ve ne sono alcune che all'apparenza sfuggono ad una immediata comprensione poiché riguardano defunti di bassa estrazione sociale. La manifestazione di quest'ultimi era tradizionalmente considerata foriera di elementi negativi, associata quasi sempre a qualche artilugio ad opera del demonio e non certo votata ad offrire quell'aiuto che solo un santo o la Vergine avrebbero potuto prestare ad un fedele ${ }^{7}$.

Uno dei casi più emblematici che evidenzia quanto appena detto è quello del ben noto "cadavere vivente" che viene presentato nella CSM 67 con tutte le modalità tipiche di una apparizione, ma che non dimostra di possedere doti angeliche, intenzioni benevole o di essere stato mandato da Dio per mettere fine a dissidi di ordine spirituale. Dietro a questa insolita manifestazione di un morto vi è infatti l'operato del demonio, il quale si impossessa momentaneamente del suo corpo - più che un revenant, dunque, è un corpo morto controllato da un altro spirito- per ingannare un uomo devoto e pio che aveva assoldato il futuro posseduto per aiutarlo in alcune opere di carità ${ }^{8}$.

I pregiudizi negativi verso questi morti ordinari ai quali viene concesso per poco tempo di agire all'interno della dimensione dei vivi si dimostrano piuttosto radicati anche nelle $C S M$, in linea con la più diffusa dottrina ecclesiastica basata sugli insegnamenti di Sant'Agostino. Quest'ultimo vedeva infatti in tali manifestazioni dell'oltretomba fenomeni di origine pagana da reprimere, scongiurare ed allontanare. Nelle CSM però, questo paradigma sembra incrinarsi proprio con il caso che si andrà ad analizzare, il quale può facilmente definirsi come un vero e proprio spaccato su un ambiente sociale che, nel corso del XIII secolo, era in processo di laicizzazione e che era arrivato a sfruttare tali credenze per legittimare la sua organizzazione in ambito laico ed ecclesiastico. Tali credenze vedevano infatti riconosciuta anche ai morti ordinari la possibilità di trasmettere messaggi positivi o di intervenire

${ }^{6}$ Questo punto di vista, poi adottato ufficialmente dalla Chiesa durante tutta l'epoca medievale, è quello che Sant'Agostino mostra chiaramente in una delle prime formulazioni della cosiddetta "teoria cristiana degli spettri": la lettera De Cura pro mortuis gerenda (421-424) indirizzata a Paolino da Nola (Schmitt 1994, pp. 34-36). Sulla funzione, da sempre riconosciuta ai santi, di entità ausiliatrici per il fedele: Vauchez 1981; 2009, pp. 447-485. Si vedano anche Le Goff 1981, pp. 111-114; Schmitt 1987, p. 37.

${ }^{7}$ Questo soprattutto nel periodo altomedievale, $c f$. Schmitt 1988b; 2013, p. 63.

${ }^{8} C f$. Martínez 1996, p. 124. Si tratta di uno dei pochi casi in cui un morto riappare sulla scena dei vivi operandovi concretamente, anche se tale entità non può essere considerata tout court come un revenant, ma come un semplice cadavere animato per negromanzia o per arte diabolica. Difatti è solo il suo corpo a rivivere per pochi istanti, essendo inoltre posseduto da un'entità estranea (in questo caso il diavolo) che si sostituisce alla sua coscienza originaria. Per un'analisi della CSM ed alcune riflessioni sul tema del living corpse, $c f$. Keller 1988-1989, p. 58; 1991, pp. 49-59. La concezione alla base di questo tipo di apparizione può essere ritrovata in una delle molteplici letture che l'esegesi biblica ha saputo fornire in merito al ben noto caso dello Spettro di Salomone. Per quest'ultimo aspetto, $c f$. Schmitt 1987, pp. 41 ss. 
nella storia dell'uomo per dare consigli, ribadire la forza o l'importanza di un lignaggio, confermare donazioni ecclesiastiche da parte dei defunti, e anche per poter prestare soccorso su mandato divino".

La storia di questa apparizione viene narrata nella CSM 72: Como o demo matou a un tafur que deostou a Santa Maria porque perdera ${ }^{10}$. In essa si racconta un singolare episodio di vendetta ordita dalla Vergine Maria ai danni di un giocatore d'azzardo che aveva non solo osato bestemmiare Dio (esibendo in tal modo la sua frustrazione per aver perso al gioco dei dadi), ma anche rivolgere parole ingiuriose nei suoi confronti. Nelle prime quattro cobras viene condensata tutta la prima parte del miracolo, tacendo però inizialmente sul tipo di punizione riservata al peccatore: si specifica soltanto come l'esecuzione del bestemmiatore fosse stata eseguita per volere di Dio, sottolineandone oltremodo gli esiti mortali (vv. 5-20) ${ }^{11}$ :

E desto quero contar miragre que quis mostrar

Deus por sa madre vingar dun mui mentiral

Quen diz mal da reĩa espirital...

que na taverna beveu

e aos dados perdeu

alg' e por en descreeu mui descomunal-

Quen diz mal da reĩa espirital...

mente, ca a Deus dẽostou

e sa madre non leixou,

e en seus nembros travou come desleal.

Quen diz mal da reĩa espirital...

E u quis do ventre seu

dizer mal, morte lle deu

Deus come a fals' encreu que de razon sal.

Quen diz mal da reĩa espirital...

\footnotetext{
${ }^{9}$ Cf. Schmitt 1987, p. 38.

${ }^{10} \mathrm{Il}$ testo appare in tre dei quattro manoscritti che trasmettono la tradizione testuale complessiva delle CSM, oltre a costituire un riflesso di diverse fasi redazionali del progetto alfonsino: To (Biblioteca Nacional de Madrid, 10069, c. 159rv), T (Biblioteca del Real Monasterio de El Escorial, T.I.1, c. 106r) ed E (Biblioteca del Real Monasterio de El Escorial, I.b.2, cc. 90v-91r). Per una sintesi sulle caratteristiche e la storia di questi tre codici, $c f$. Fidalgo 2002a, pp. 51-58. Per informazioni più dettagliate, $c f$. Schaffer 1999; Fernández Fernández 2008-2009.

${ }^{11}$ Per l'organizzazione metrica e strofica del testo qui riprodotto, si tiene conto di quanto proposto in Parkinson 2010, pp. 324-329. Quanto al testo critico, se ne condividono in linea generale le scelte editoriali apparse nella sua più recente edizione (Parkinson 2015, pp. 66-67), dalla quale si cita, sebbene introducendo un sistema di numerazione progressivo per i versi riprodotti (nell'edizione, infatti, la numerazione riparte per ogni strofa). Per un confronto col testo offerto dall'edizione critica complessiva delle CSM considerata ancora come di riferimento, si rimanda comunque a Mettmann 1986, pp. 238-240.
} 
La storia prosegue poi con l'entrata in scena del padre del peccatore. Non appena riceve infatti la notizia della morte del figlio (quand' est' oiu, v. 21), si precipita in strada per recarsi sul luogo del misfatto. Ma ecco che nel bel mezzo del sentiero che stava percorrendo gli si manifesta davanti, senza alcun preavviso, quello che nella CSM viene definito un morto (...) ben di natural (vv. 21-24):

Seu padre, quand' est' oiu, de sa cas' enton saiu, na via un morto viu ben di natural, Quen diz mal da reĩa espirital...

Si tratta proprio dello spettro già menzionato, che in seguito inizia a parlare per giustificare le ragioni della sua improvvisa comparsa (atal razon, v. 25), consegnando al padre del peccatore un duplice messaggio. Dapprima allega le ragioni della morte del figlio: a detta del fantasma, così come già descritto nelle cobras precedenti, il ragazzo avrebbe meritato la punizione celeste non solo per aver bestemmiato direttamente Dio, ma anche per aver mancato di rispetto a sua Madre (si intenda, la Vergine Maria), arrivando addirittura a schernire il suo ventre benedetto (vv. 25-32) ${ }^{12}$ :

que lle diss' atal razon:

"Teu fillo, mui mal garçon, é mort' e en perdiçon, que nunca mais fal, Quen diz mal da reĩa espirital...

non porque de Nostro Sennor

disse mal, mais que da flor, sa madre, disse peior (...)

Quen diz mal da reĩa espirital...

\footnotetext{
${ }^{12} \mathrm{Nel}$ testo si legge "quis do ventre seu / dizer mal", ovvero "volle parlar male del suo ventre'. Ariel Guiance, trattando i casi di fantasmi nelle CSM, offriva qui una diversa interpretazione, identificando il ventre danneggiato con quello del protagonista (laceratosi in seguito alle bestemmie proferite); $c f$. Guiance 1998, p. 405. Più verosimilmente, qui il poeta sembrerebbe far riferimento al ventre della Vergine (ovvero Cristo), schernito ed offeso dal giocatore. Quest'ultimo infatti morirà a causa di ferite esteriori ben più gravi. Se proprio si volesse identificare il ventre seu con l'addome del gambler, allora si potrebbe considerare il ventre nel suo valore di complemento di luogo figurato da dove sarebbero fuoriuscite, in una sorta di fisiologia della blasfemia, le parole ingiuriose, ovvero dalle stesse viscere, sede tradizionalmente deputata ai più bassi ed irrazionali istinti dell'uomo. L'appellativo "Madre di Dio" è diretta conseguenza della emanazione del principio della theotókos. All'indomani del Concilio di Efeso del 413, Maria può infatti essere ritenuta a tutti gli effetti madre dell'unico Dio in quanto in essa Dio stesso si era incarnato per nascere tra i mortali. Ritroviamo questo concetto espresso nelle CSM svariate volte proprio attraverso l'adozione di tale appellativo; $c f$. Fidalgo 2002b, p. 89; Alcalá 1996, p. 35.
} 
Nella seconda parte del messaggio, l'anonima entità rivela all'uomo alcuni dettagli utili per il riconoscimento del cadavere. Si apprendono così finalmente dalle sue parole gli effetti fisici della punizione divina, eseguita attraverso il braccio del demonio (come indicano in modo chiaro le diverse rubriche nei manoscritti): il padre potrà riconoscere il corpo -e così provvedere a dargli sepoltura- per il fatto di presentare un'orribile mutilazione giusto al mezzo, come se fosse stato colpito da una specie di saetta. La CSM si conclude senza ulteriori colpi di scena: il padre arriva sul luogo dove si trovano le spoglie del figlio, le riconosce e le prende con sé (vv. 32-45):

e por en sinal

Quen diz mal da reĩa espirital...

ti dou, que o acharás

pelas costas tod' atras

partid', e ll' o cor verás assi per igual

Quen diz mal da reĩa espirital...

a testa e a serviz,

porque da Emperadriz

disse mal, Deus foi joiz que pod' e que val".

Quen diz mal da rẽ̃a espirital...

O padre foi log' ali

e achou seu fill' assi

como vos ja retraí, ben oistes qual.

Quen diz mal da reĩa espirital...

\section{UN’APPARIZIONE NON SEMPRE 'TRASPARENTE’: LETTURE PRECEDENTI}

Sul motivo miracolistico presente nella CSM 72, ossia quello dell'apparizione di una figura diversa dalla Vergine Maria o di un santo dall'Aldilà cristiano per fornire un aiuto ad un mortale, la critica non si è sempre espressa con cognizione di causa. In più, le informazioni sulla sua natura, la sua identità e l'interpretazione del ruolo che riveste nel racconto non hanno mai ricevuto un approfondimento capace di portare ad una chiara decodifica di questo exemplum mariano.

In uno studio che prendeva in esame questa narrazione miracolistica nel contesto delle CSM dei giocatori d'azzardo puniti da Dio e dalla Vergine, Dwayne E. Carpenter offriva per primo qualche suggerimento sull'identità del fantasma. Nel sottolineare le punizioni divine inflitte a chi si dilettava in questo tipo di attività ludiche nelle CSM (prova della stessa ostilità che Alfonso $\mathrm{X}$ nutriva nei loro confronti e delle limitazioni giuridiche fatte vergare 
per ostacolarne la proliferazione) $)^{13}$, lo studioso sembrava escludere l'ipotesi che si dovesse identificare il protagonista di questa apparition con lo stesso peccatore morto da poco tempo. Scriveva a tal riguardo: God himself kills a lying, drinking, blaspheming gambler, and then, through an apparition, informs his father that he will find the body of his wretched son split asunder ${ }^{14}$. Carpenter non aggiungeva nient'altro per avallare questa sua lettura e -cosa altrettanto rilevante- non prendeva nemmeno in considerazione l'ipotesi di una conoscenza in vitam tra l'uomo e questo inusuale messaggero divino, che sembrava quindi non essersi manifestato a seguito di un'iniziativa del tutto personale, ma come strumento nelle mani di Dio per agevolare il ritrovamento delle spoglie del ragazzo.

Ancor prima della lettura di Carpenter, John E. Keller e Richard P. Kinkade avevano percepito in modo analogo l'identità e il ruolo del suddetto fantasma, nonché le ragioni del suo agire nella seconda parte della narrazione. Volendo infatti descrivere alcuni supernatural beings ravvisabili nei testi mariani alfonsini attraverso l'analisi del programma iconografico del Códice Rico, così i due commentavano la scena della famosa apparizione raffigurata nel quarto riquadro della c. $106 \mathrm{v}^{15}$, coincidente punto per punto con i versi centrali della CSM 72: (...) a ghost in a winding sheet, just as it might appear in art today, manifests to a man that his son is dead and in the other world ${ }^{16}$. Ancora una volta dunque, dopo una breve descrizione esteriore dello spettro e i richiami ad una sorta di modernità nella sua raffigurazione, il ghost non veniva ricondotto al peccatore morto: per Keller e Kinkade si sarebbe potuto trattare solamente di un messaggero dell'oltretomba cristiano, apparso sul

${ }^{13}$ Si ricordi, infatti, come nella Settima Partida (Titolo XIV, Legge VI) Alfonso apostrofi i giocatori d'azzardo in malo modo e ne condanni il passatempo, sulla base di due ragioni: l'attività illecita di guadagno che sarebbe potuta derivare dalla vincita del gioco e il rischio, sempre presente, che l'attività ludica se non regolamentata potesse portare al riscaldamento degli animi e dunque anche ad esternazioni blasfeme. Nel 1276, a ribadire la sua posizione di ferma condanna, il saggio re castigliano emanerà il celebre Ordenamiento de las tafurerias con il quale "por ninguna manera" permetterà il gioco d'azzardo, $c f$. Fournès 2015. Sulla degenerazione che il gioco dei dadi poteva comportare, fino agli esiti della bestemmia, con un'interessante profilo per l'area italiana sulle misure contenitive che vennero prese in diversi comuni, $c f$. Moretti 2001, pp. 109-119.

${ }^{14}$ Carpenter 1998, p. 344 . Precedentemente, in un altro contributo consacrato allo studio delle reazioni vendicative della Vergine nei confronti di alcuni peccatori, come quelli che mettevano in atto un vero e proprio affronto nei suoi riguardi attraverso il ricorso al turpiloquio, William R. Davis menzionava la stessa CSM 72, ma senza ma senza descriverla in modo particolareggiato; $c f$. Davis 1974, p. 99.

${ }^{15}$ Per un riscontro visuale, si rimanda all'edizione facsimilare Fernández Fernández 2011. Come è noto, il ms. Biblioteca del Real Monasterio de El Escorial, T-I-1 (T) è l'unico a presentare un vasto programma iconografico per alcune delle CSM che trasmette, assieme al ms. Biblioteca Nazionale Centrale di Firenze, Banco Rari 20 (F).

${ }^{16}$ Keller, Kinkade 1984, p. 11. 
posto in qualità di aiutante divino, ma del tutto estraneo agli eventi da poco occorsi e alla famiglia del ragazzo.

A queste postille sono seguiti contributi più articolati, utili per le riflessioni che verranno presentate al termine di questo lavoro, come quello ad opera di Jean-Claude Schmitt nell'ambito di una monografia divenuta ormai imprescindibile per lo studio delle manifestazioni e delle percezioni soprannaturali nel medioevo: Les Revenants: les vivants et les morts dans la société médiévale. Il medievista francese classificava questo componimento come una delle due histoires de revenants presenti nel corpus mariano alfonsino, dedicando ampio spazio alla resa figurativa dei fantasmi nel Códice Rico ${ }^{17}$. Se per la prima narrazione miracolistica che faceva rientrare in tale categoria (CSM 123) e a partire dall'esame della quinta vignetta che ne illustra la presenza soprannaturale che opera nella storia, Schmitt sottolineava la difficoltà di riconoscere un morto nella figura che viene rappresentata all'interno di un chiostro se non ci si aiuta col testo ${ }^{18}$, affermava invece come per la CSM 72 l'individuazione di quello che lui chiamava revenant fosse del tutto immediata. Accorrevano infatti in ausilio del lettore dei precisi stilemi formali che lo differenziavano dalla figura di maggiore densità cromatica che gli stava accanto: pallidezza, diafanità degli abiti, bianchezza, ecc. Inoltre, sempre a detta del medievista francese, la sua figurazione più eterea avrebbe potuto suggerire un decesso avvenuto in un passato più remoto rispetto a quello del morto ancora pienamente "fisico" della CSM 123, oltre a rivelare -sempre grazie all'espediente figurativo della sua trasparenza- una condizione di estraneità tra questi e il padre del ragazzo. Per il resto, quanto all'identità dello spettro, veniva riproposta l'ipotesi precedente: non già le jeune homme qui vient de mourir, bensì un anonimo messager de l'au-delà et de la Vierge.

Ariel Guiance, in un capitolo dedicato all'analisi dei fantasmi nelle CSM, riprendeva da vicino le posizioni di Schmitt aderendo anche alle ipotesi sull'identità spettrale, sebbene sulla sua funzione e sul suo vincolo coi protagonisti si mostrasse incline a limitarne la sfera di influenza: el aparecido sólo es un mensajero que anuncia al padre (...) la dureza de la pena impuesta a su hijo. Entre el fantasma y el testigo no existe mayor vinculación ${ }^{19}$. Ma la parte più interessante della sua indagine era quella che proponeva per il personaggio lo status di morto ordinario ancora privo della grazia divina, dedotto dalla

\footnotetext{
${ }^{17}$ Schmitt 1994, pp. 239-241. L'altra, la CSM 123, narra la storia di un frate francescano che viene soccorso e protetto dalla stessa Vergine Maria in punto di morte. Quest'ultima era intervenuta per impedire che un gruppo di diavoli riuscisse ad impossessarsi della sua anima negandogli pertanto la possibilità di accedere al Regno dei Cieli.

${ }^{18}$ Osservava infatti come il frate che era apparso nel chiostro ai suoi confratelli non si distinguesse da quest'ultimi "ni par l'apparence corporelle ni par l'habit", $c f$. Schmitt 1994, p. 240.

${ }^{19}$ Guiance 1998, pp. 405-406.
} 
peculiarità iconografica già notata da Schmitt -la quasi assoluta trasparenzarispetto al fantasma del monaco della CSM 123 raffigurato a piena tinta, e per questo già pienamente partecipante della visione celeste proprio in virtù di questa sua riconoscibilità da parte di alcuni uomini di chiesa ${ }^{20}$.

Alla luce di queste letture più o meno convergenti, sorgono e in parte si ripropongono alcune domande in merito alla CSM e al suo fantasma. Si tratta veramente di una imago estranea al padre del ragazzo? E sulla base di quali credenze o dinamiche implicite tale personaggio può manifestarsi rivestendo il suo ruolo di armier $^{21}$ dell'aldilà cristiano ${ }^{22}$, nel sostituirsi dunque a Maria e ai santi nell'esercizio delle loro tradizionali funzioni di intermediazione e di ausilio per il fedele di turno? L'obiettivo del lavoro sarà quello di dare una risposta più circostanziata alle questioni ancora in sospeso, partendo questa volta da un confronto serrato tra la CSM, le sue possibili fonti scritte e l'ambiente culturale nel quale i testi sono stati creati e promossi.

\section{UNA RIVELAZIONE NELLA TRADIZIONE}

Come spesso accade per un'opera di particolare complessità come lo sono le CSM, nella quale la densità semantica dei testi è data dall'apportazione di una varia tipologia di fonti, il ricorso a quest'ultime si rivela fondamentale anche per fornire risposte più argomentate sul 'fantasma' oggetto di studio, illuminandone così gli aspetti non espressi o che si è scelto -volontariamente o meno- di non includere tra i versi.

Non si vuole, con questo, scorgervi intenzioni narrative che vadano al di là di quanto poco ci viene detto o illustrato nella CSM 72 (sicuramente non era nemmeno intenzione del Rei Sabio approfondirne il ritratto nella storia, dove svolge una funzione del tutto ancillare al servizio di una dimostrazione di misericordia estrema, garantita dalla Vergine anche ai peccatori più recidivi) ${ }^{23}$. Quello che si vuole sottolineare è unicamente il percorso culturale

\footnotetext{
${ }^{20}$ Ibidem, p. 408. Tale osservazione, che sviluppa il discorso già intavolato da Jean-Claude Schmitt, è a nostro avviso fondamentale per decodificare in maniera più chiara le ragioni della presenza di questa entità nel mondo dei vivi della CSM alfonsina. Verrà ripresa al termine del contributo per ulteriori precisazioni.

${ }^{21}$ Sull'armier, intermediario tra vivi e morti delle campagne occitane, $c f$. Martínez 1996 p. 123.

${ }^{22} \mathrm{Col}$ termine imago ci si rifà alla dottrina cristiana in voga a partire dal XII sec., quando probabilmente Alchero di Chiaravalle, recuperando e rivisitando la dottrina di Sant'Agostino sulle manifestazioni dall'Aldilà cristiano, ritiene la categoria del phantasma (o phantasmata) prodotto della visio spiritualis comune agli individui, la quale permette di vedere non dei corpi, ma delle loro apparenze.

${ }^{23}$ Cf. França 1999.
} 
che ne ha permesso la sopravvivenza fin nello stesso exemplum mariano, nonché quali idee sull'Aldilà cristiano ne avrebbero potuto legittimare in via progressiva quell'azione d'intervento che, vagliata da una larghissima tradizione religiosa e culturale, è arrivata a rinnovarsi nelle pagine del Re Sapiente ${ }^{24}$.

\subsection{La cantiga 72 e la tradizione dello Speculum Historiale}

Il prodigio versificato da Alfonso X e il suo entourage appartiene ad una nutrita famiglia di miracula virginis riconducibili al ben noto canovaccio narrativo del "giocatore punito" dalle alte sfere in seguito alle bestemmie proferite senza ritegno per aver perso al gioco dei dadi. Il motivo viene registrato anche nell'Index Miraculorum di Tubach con alcune varianti. Si rimanda, a tal proposito, al $\mathrm{n}^{\circ} 680$ (Blasphemer, struck by lighting. The son of a knight is killed by a thunderbolt because his father cursed the ban weather) che evidenzia un tipo di punizione alternativa per il peccatore, $\mathrm{o}$ al $\mathrm{n}^{\circ} 2240$, nel quale si può far rientrare anche il caso della CSM 72: Gambler, swearing of. A gambler, losing in a dice game, dies suddenly when he blasphemes ${ }^{25}$.

Si tratta di un tema che ha conosciuto una fortuna notevole tra i miracoli di Maria e la cui circolazione, certamente importante anche nel periodo in cui opera il Re Saggio, è testimoniata dal buon numero di declinazioni narrative assunte in altrettante collezioni di miracoli anonime o di autore noto, disseminate su tutto il territorio europeo ${ }^{26}$.

Il censimento effettuato per l'occasione ha portato ad individuare più di una ventina di raccolte di miracula virginis che, all'interno di questa grande famiglia, ospitano il motivo in una veste che ricorda quella della CSM 72, indizio di una presenza certamente più nutrita in altre collezioni che, ad oggi, non si sono ancora potute consultare o localizzare ${ }^{27}$. Si tratta perlopiù di opere

\footnotetext{
${ }^{24}$ In altre parole, alla pari di quanto proposto recentemente da Nancy Mandeville Caciola prendendo come base i racconti del vescovo Thietmar of Merseburg (1013-1018), si vuole qui utilizzare il testo alfonsino assieme alle fonti culturali e letterarie che avrebbero contribuito alla sua costruzione per definire quali credenze sulle apparizioni fantasmagoriche sarebbero potute approdare alla corte del Re Sapiente; le stesse che avrebbero permesso non solo una adozione del récit, ma anche di percepirlo come legittimo nel contesto miracolistico mariano; $c f$. Caciola 2014.

${ }^{25} C f$. Tubach 1981, pp. 56, 179. $C f$. anche ThEMA.

${ }^{26}$ Come è risaputo, il primo gruppo di cento CSM proviene per la maggior parte da collezioni di carattere universale, non legate cioè all'attività propagandistica di un santuario locale; cf. Parkinson 2011. Da questo punto di vista, la CSM 72 non fa eccezione.

${ }^{27}$ Oltre alla variante qui presa in esame, ve ne sono altre che mostrano una gran proliferazione delle tipologie di punizione divina messe a punto dalla fantasia monastica a seguito del peccato commesso. Si ricordi solamente uno degli exempla del domenicano Stefano di Borbone che racconta come un giocatore della diocesi di Rheims bestemmiasse Dio mentre giocava ai dadi nel giorno della Cena del Signore. La punizione che ne segue è strabiliante: caduto in preda
} 
compilate tra il XII e il XV sec. in area francese e soprattutto inglese ${ }^{28}$. Successivamente, il tema sembrerebbe aver conosciuto un trattamento maggiore nell'area mediterranea, nella fattispecie italiana ${ }^{29}$, in accordo con le direttrici di sviluppo e diffusione della letteratura miracolistica.

Nella tabella a continuazione, vengono raccolti i risultati di questa indagine. Per ogni collezione di miracoli mariani, si riportano nelle colonne quarta e quinta le informazioni per localizzare le narrazioni in esse contenute. Si indica inoltre il periodo a cui risale la compilazione di alcune raccolte e, nel caso in cui la stessa collezione si riveli adespota, quello della produzione di alcuni manoscritti che offrono la testimonianza di vari nuclei miracolistici mariani o già proto-raccolte fissatesi nella tradizione ${ }^{30}$ :

\begin{tabular}{|l|l|l|l|l|l|}
\hline $\mathrm{N}^{\mathrm{o}}$ & \multicolumn{1}{|c|}{ AUTORE } & \multicolumn{1}{c|}{ COLl./MS. } & \multicolumn{1}{c|}{ CARTE } & \multicolumn{1}{c|}{ CAPITOLO } & \multicolumn{1}{c|}{ PERIODO } \\
\hline 1 & Anon. & Par. lat. 3177 & $118 \mathrm{v}-119 \mathrm{v}$ & $/$ & XII sec. \\
\hline 2 & C. de Heisterbach & Dial. Miraculorum & $/$ & VII, 43 & Inizi XIII sec. \\
\hline 3 & V. de Beauvais & Spec. Historiale & $/$ & VIII, 104 & Ante 1260 \\
\hline 4 & Anon. & Add. 15793 & $79 \mathrm{v}$ & 18 & XIII sec. \\
\hline 5 & Anon. & Add. 32248 & $/$ & 3 & XIII sec. \\
\hline 6 & $\begin{array}{l}\text { Étienne de } \\
\text { Bourbon }\end{array}$ & Tractatus & $/$ & $112,113,387$, & XIII sec. \\
\hline 7 & Anon. & Egerton 1117 & $182 \mathrm{r}$ & 15 & Fine XIII sec. \\
\hline 8 & Anon. & Arundel 506 & $4 \mathrm{r}$ & 17 & Inizi XIV sec. \\
\hline 9 & Anon. & Add. 18346 & $33 \mathrm{v}$ & 117 & XIV sec. \\
\hline
\end{tabular}

ad una sorta di attacco epilettico, l'uomo iniziò a fagocitare il suo stesso corpo, partendo dagli arti, tanto da rimpicciolirsi fino ad assumere le sembianze di un bambino; $c f$. Moretti 2001 , p. 118. Oltre a basarci su studi anteriori, fondamentale per l'individuazione delle collezioni che si citeranno è stata la consultazione di Poncelet 1902.

${ }^{28}$ Il caso più emblematico è quello del ms. London, British Library, Additional 15723 che dà conto di una narrazione simile alla CSM 72 raccolta e compilata in territorio inglese nel XIII sec.. Tale raccolta era stata già censita in relazione alla CSM 72 anche in Forsythe 1926, p. 126. Sul codice, $c f$. Ward 1893, pp. 628-629.

${ }^{29} \mathrm{Ci}$ si riferisce alle grandi sillogi di produzione tardomedievale che compaiono nella Penisola, ma che potrebbero comunque essere state precedute da testimoni anteriori, oggi non noti. Il tema del "bestemmiatore ucciso" compare nelle collezioni del Libro del Naufragio e del Libro del Cavaliere, ma solo nel miracolo $\mathrm{n}^{\circ} 99$ del secondo il protagonista è effettivamente un giocatore d'azzardo, richiamando dunque più da vicino la CSM 72; cf. Gripkey 1952.

${ }^{30}$ Per una breve disamina di questi nuclei o raccolte primitive dedicate ai prodigi mariani su scala europea, poi riproposte in testimoni più tardi, integrate con altre o aggiornate con nuovi prodigi, si rimanda alla sempre utile consultazione di Faye 1946, pp. 3-77. Oltre al lavoro appena menzionato, le varie voci della tavola vengono riunite e compilate a partire dai dati già raccolti in Parkinson 2005-. 


\begin{tabular}{|l|l|l|l|l|l|}
\hline 10 & Anon. & Add. 18364 & $13 \mathrm{v}$ & 24 & XIV sec. \\
\hline 11 & Broun (?) & Add. 33956 & $5 \mathrm{r}$ & 3 & XIV sec. \\
\hline 12 & Jean Gobi & Scala coeli & $/$ & XII, 1 & XIV sec. \\
\hline 13 & Jean de Vignay & Miroir Historial & $/$ & VIII, 104 & XIV sec. \\
\hline 14 & Anon. & Libro del Naufragio & (vari mss.) & 16 & XIV-XV sec. \\
\hline 15 & Anon. & Libro del Cavaliere & (vari mss.) & $23,39,99$ & XIV-XV sec. \\
\hline
\end{tabular}

Se il motivo principale che vede il giocatore (o un altro protagonista) ricevere una punizione mortale ricorrendo a modalità simili a quelle descritte nella CSM 72 si ravvisa in un buon numero di exempla, l'aggiunta della sequenza in cui appare ed opera il fantasma per agevolare il ritrovamento del cadavere riguarda solamente tre casi (si tratta di quelli evidenziati nella tabella precedente). Tutti e tre si rifanno inoltre ad uno stesso récit. Il primo è quello che fa capo alla collezione anonima trasmessa dal ms. Paris, Bibliothèque nationale de France, lat. 3177 alle cc. $118 \mathrm{v}-119 \mathrm{v}$ e che, secondo parte della critica, potrebbe trasmettere quel famoso Mariale Magnum nominato con frequenza dal domenicano francese Vincent de Beauvais come una delle fonti principali utilizzate per la confezione del suo Speculum Historiale ${ }^{31}$. Il secondo caso è proprio quello contenuto in quest'ultima opera di grandissima fortuna, la cui compilazione precede perlomeno il $1260^{32}$. Il terzo rinvenimento è posteriore agli altri due e anche all'opera del Re Sapiente, essendo stato di fatto inglobato nella traduzione francese dello Speculum Historiale a cura di Jean de Vignay: si tratta del Miroir Historial, composto durante le prime due decadi del XIV $\sec ^{33}$.

Le prime due occorrenze sono dunque le sole a trasmettere una narrazione degli eventi che, sebbene in prosa, potrebbe essere stata la fonte di ispirazione per Alfonso X e i suoi collaboratori, almeno durante la fase di raccolta del materiale per la CSM 72, prima di procedere alla sua versificazione $^{34}$. In generale, tenuto conto dell'importanza e la presenza dello Specu-

\footnotetext{
${ }^{31}$ Considerando sia il periodo di produzione del testimone sia la data successiva di elaborazione dello Speculum con la quasi totale sovrapponibilità dei due récits, l'ipotesi si rivela del tutto plausibile. Si rimanda alla consultazione di Marchand 1998. Sul Mariale Magnum, $c f$. Barre 1966. Su Vincent, $c f$. Paulmier-Foucart 2004. Sull'utilizzo del Mariale Magnum da parte di Vincent, $c f$. Voorbij 1997; Paulmier-Foucart 2004, pp. 84-87.

${ }^{32}$ Ma probabilmente anche il 1254. Sulla datazione delle differenti tappe della compilazione dello Speculum, con particolare attenzione alla sezione che dimostra i debiti maggiori col Mariale Magnum più volte citato fra le fonti, $c f$. Voorbij 1990.

${ }^{33}$ Si ravvisa una dinamica simile nel motivo ${ }^{\circ} 684$ in Tubach 1981, dove un bambino di cinque anni viene condannato per blasfemia davanti al padre. Procede dai Dialogi di Gregorio Magno, ed è forse il più famoso in merito alla relazione padre-figlio blasfemo, con annessa apparizione dall'oltretomba.

${ }^{34}$ Sui vari momenti che interessano la produzione delle CSM, $c f$. Parkinson, Jackson 2006.
} 
lum di Vincent tra le fonti delle CSM, si può ipotizzare con un certo grado di sicurezza come la narrazione del domenicano fosse quella materialmente presente sul tavolo di lavoro del re castigliano-leonese. La conoscenza e la fruzione dell'opera del domenicano francese da parte di Alfonso X è molto probabile: oltre a tener presente come lo Speculum historiale abbia avuto un ruolo di primo piano per tutta la produzione del Sabio, come, ad esempio, la compilazione della Estoria de España, si è a conoscenza infatti della presenza di una copia fisica dello Speculum nell'armadium del sovrano (probabile dono del cugino Luigi IX) attorno agli anni '60 o "70 del sec. XIII' ${ }^{35}$. Anche una sua disamina interna non può che confermare quanto appena ipotizzato, dato che le varie sequenze narrative combaciano alla perfezione con quanto raccontato in versi nella CSM alfonsina poco tempo dopo ${ }^{36}$ :

Huius pater fama excitatus veniebat ut filio suo lamentabiles exequias exhiberet. Cui in itinere quidam vicinus suus, paulo ante mortuus, occurrit et ait: Durum tibi apporto nuncium quia filius tuus sempiterna morte mortuus est, quia Christum blasphemavit et matri eius detraxit. Et hoc tibi signum. Invenies cadaver eius a cervice usque ad nates per mediam dorsi spinam quasi sectum et cor in duas partes dissiluisse tabefactum. Hoc dicto disparuit, et pater pergens hec signa invenit.

Si può però osservare come il récit del domenicano si allontani, in alcune occasioni, dalla brevitas estrema ricercata dai versi del testo del Re Sapiente, finendo per trasmettere informazioni più estese in merito ad alcuni aspetti. Uno di questi riguarda proprio i dettagli sull'identità dello spettro, presentato da Vincent de Beauvais come un vicinus del padre del peccatore morto: in altre parole, un suo 'compaesano'. Si veda anche, a tal proposito, il volgarizzamento a cura di Jean de Vignay messo a punto tra il 1293 e il $1349^{37}$ e che ricalca punto per punto il precedente testo latino:

${ }^{35}$ Forse anche grazie all'intermediazione di Juan Gil de Zamora, studente di teologia a Parigi tra il 1267 e il 1277 . Sulla copia dello Speculum posseduta da Alfonso X, $c f$. Domínguez 1998, p. 173; Rubio 1985. Per uno studio più recente, a riconferma dell'influenza esercitata da Vincent sull'opera mariana del Sabio, $c f$. Taylor 2015.

${ }^{36} \mathrm{Si}$ cita il testo dall'edizione digitale della versione del Ms. Douai BM 797 presente in Sources des encyclopédies médiévales (SourcEncyMe). Il testo può essere recuperato a partire dal menù che mostra la suddivisione in capitoli dello Speculum. Uno dei segnali di una operazione di trasposizione dal testo latino al nuovo sistema linguistico galego-portoghese spesso condotta "verbo a verbo", può essere, ad esempio, il calco na via a partire dal sintagma latino in itinere, quando si precisa la posizione del genitore nell'istante in cui quest'ultimo riceve la visita inaspettata del fantasma.

${ }^{37} \mathrm{Si}$ cita da Cousteau, et al. 1531, p. 470. Per facilitare la lettura, vengono sciolte le abbreviazioni mantenute nell'edizione a stampa in grafia corsiva. 
Et le pere de celluy est meu pour la renommee et venoit pour faire a son filz ploreu ses evecques ung sien voysin qui estoit mort ung peu avant luy acourut au devant et dist. Je tapporte un dur message: car ton filz est mort de mort pardurable pource quil blasma Jesuchrist $e t$ vitupera la Vierge Marie sa mere et tu trouveras ce signe car la charogne de luy tu trouveras trenchee depuis le hault de la teste (...) Et quant il eut ceste chose dicte il se desapparut. Et le pere allant la trouva ces signes.

Questo dato, se da una parte può dunque già allontanare l'ipotesi che anche nelle intenzioni di Alfonso X e del suo entourage il fantasma dovesse essere identificato con una imago del ragazzo morto (riconfermando dunque le impressioni degli studiosi che si sono pronunciati su questo punto), dall' altra ci consente di interpretare in modo più sicuro l'aggettivo natural che viene associato al morto nella CSM 72 (v. 23): non si deve infatti pensare che si tratti di una qualifica tesa a caratterizzare la sua parvenza esteriore o una sorta di conformità con la natura del mondo circostante (un morto che sembrava quasi vivo), ma un riferimento preciso al suo essere originario di quel luogo ${ }^{38}$.

Nelle CSM si possono trovare vari esempi dell'aggettivo natural col valore di "conterraneo, nato in quella terra, abitante del luogo". Tra i casi più espliciti che affiancano all'aggettivo anche un complemento o un avverbio di luogo a conferma di tale possibilità di significato (accanto a quelli di "conforme alla natura, normale" o "legittimo, legale"), si ricordino, a titolo di esempio, il passo della CSM 15 che informa sulla provenienza del filosofo Libano (filosofo natural de Sur, v. 161), o quello del privado di Tolosa nella CSM 78 (enviou outr' ome natural de Tolosa, v. 58) ${ }^{39}$. Anche nel contesto della lirica profana galego-portoghese, il valore di natural come "abitante del luogo" può riconoscersi in una manciata di testi, tra i quali: 18:3 Ansur Moniz, muit'ouve gran pesar di Alfonso X (a d'Azeved'ar é mui natural, / u jaz seu padr' e sa madr' outro tal, vv. 12-13); 116:29 Quand' eu un dia fui en Compostela di Pedr'Amigo de Sevilha (e, pois for a romaria acabada, / aqui, d'u sõo natural, do Sar, vv. 33-34) $)^{40}$.

Tornando ai precedenti della CSM 72, inutile dire che anche la presunta fonte di Vincent, quella cioè contenuta nel corposo ms. Paris, Bi-

\footnotetext{
${ }^{38}$ Anche la traduzione inglese nella più recente proposta critica a cura di Stephen Parkinson recupera giustamente il valore dell'aggettivo, scegliendo il termine neighbour; $c f$. Parkinson 2015, p. 67.

${ }^{39} \mathrm{Cf}$. Mettmann 1986, pp. 98, 254.

${ }^{40}$ Per la ricerca terminologica, $c f$. Ferreiro 2018-; Brea 2018-. Per gli incipit e i versi dei due componimenti citati, si rimanda, nell'ordine, alle edizioni critiche di riferimento: Paredes 2010 , p. 204; Marroni 1968, p. 253. Per agevolare la lettura, la veste grafica dei versi citati è stata normalizzata in base ai criteri esposti in Ferreiro, et al. 2007.
} 
bliothèque nationale de France, lat. 3177, trasmette la stessa informazione (c. $119 \mathrm{r})^{41}$ :

Sed et pater eius fama excitus veniebat. ut filio suo lamentabiles exequias exhiberet. Cui cum adhuc in itinere positus esset. quidam vicin $u$ s suus qui paulo ante mortuus fuerat occurrit eum que se bene agnoscentem. et pro rei novitate stupentem ne timeret hortatus.

Proseguendo però nella lettura delle due fonti, salta all'occhio un'ulteriore informazione sull'aparecido, del tutto assente nel testo della CSM 72. Essa ha a che vedere con le circostanze temporali della morte di questa entità: paulo ante mortuus (fuerat). Vincent e l'anonimo del ms. Paris, Bibliothèque nationale de France, lat. 3177 sentono cioè il bisogno di specificare come il fantasma del vicino del padre fosse morto solo 'poco tempo prima' del figlio di quest'ultimo.

Se tale inciso narrativo, da un lato, ci consente ora di riconfermare in modo inequivocabile come il giocatore e il fantasma siano due entità completamente distinte in quella sorta di proto-exemplum che trascende le realizzazioni latina, francese e galego-portoghese (evidenziando a questo punto la funzione generale di messaggero divino assunta dallo spettro) ${ }^{42}$, dall'altro lato si rivela capace di richiamare in blocco tutta una serie di credenze che, codificate ed organizzate in altrettante narrazioni edificanti che trattano vicende di spettri, costituiscono degli assiomi fondamentali che regolano le possibilità e le finalità della manifestazione di queste imago nel corso del Basso Medioevo non molto tempo dopo la loro dipartita.

Si tratta di principi che, in un certo senso, influiscono anche sulle stesse dinamiche di tali racconti e che si possono ritrovare condensati in costanti narrative alle quali pure questo exemplum mariano dimostra di ricorrere per rendere credibile, accettabile e culturalmente familiare quanto viene raccontato $^{43}$. Tuttavia, ancora sfugge qualche elemento in merito alla missione

\footnotetext{
${ }^{41} \mathrm{Si}$ trascrive il testo direttamente dall'edizione digitale fotografica accessibile dal database Gallica. Per la sua trascrizione, considerata ancora l'assenza di un'edizione critica, si preferisce mantenere visibile lo scioglimento delle abbreviazioni e dei compendi ricorrendo alla lettera corsiva. L'exemplum mariano è registrato col n ${ }^{\circ} 29$ all'interno del secondo libro della raccolta. Alcuni dati sommari su quest'ultima possono essere ricavati da Parkinson 2005.

${ }^{42} \mathrm{Il}$ proto-exemplum può intendersi come un archetipo narrativo qui comune ad Alfonso e a Vincent, proprio in quanto il modello latino per Alfonso è lo stesso Speculum. Per questo concetto, si rimanda a Delcorno 1989, p. 164.

${ }^{43} \mathrm{Su}$ queste credenze di dominio generale, si ricordi quanto scriveva a propósito Fernando Martínez Gil: "Durante varios siglos convivieron con el cristianismo creencias y prácticas basadas en la imbricación de los muertos en el mundo de los vivos. Ante un cielo y un infierno imprecisos y relegados a los tiempos posteriores al juicio, ante la ausencia de un lugar interme-
} 
dello spettro: la sua finalità si esaurirebbe nella consegna di un messaggio o ci potrebbe essere anche dell'altro che possa spiegare la sua presenza e che la CSM 72 ormai lascia del tutto inespresso? Un esempio può essere d'aiuto per poter cambiare la nostra prospettiva di osservazione in merito a questo $\mathrm{mi}$ raculum e per comprendere ancor di più la presenza di un 'fantasma' in una storia come questa.

\subsection{Il modello 'petrino' sullo sfondo}

Tra le molteplici storie di spettri progressivamente diffusesi a partire dai secoli centrali del Medioevo ${ }^{44}$, spiccano quelle contenute nella raccolta latina De Miraculis Libri Duo, compilata tra il 1145 e il 1157 da Pietro il Venerabile, abate di Cluny ${ }^{45}$. Uno dei prodigi che la compongono può rivelarsi utile non solo per spalancare del tutto la finestra su quegli aspetti culturali che il confronto tra la CSM 72 e le sue fonti narrative hanno già contribuito in parte a disvelare, come la possibilità di manifestazione vincolata ad una originaria interruzione inattesa della vita della persona ${ }^{46} \mathrm{e}$ il permesso di realizzarla solamente in un momento cronologicamente vicino al suo decesso ${ }^{47}$, ma anche per ragionare sulle finalità (nascoste) dell'apparizione del fantasma. Queste, infatti, erano quasi sempre da ricondursi alla richiesta di suffragi ai vivi piú prossimi per parentela o vicinanza spaziale e/o spirituale per poter uscire dal Purgatorio ${ }^{48}$.

dio, los muertos vagaban buscando un lugar de reposo, conversaban entre sí, se manifestaban a los vivos para que se pagasen sus deudas o reparasen sus errores" (Martínez 1996, pp. 122-123).

${ }^{44} \mathrm{Si}$ veda, per tali periodizzazioni che danno conto dell'iniziale ostilità della Chiesa nei confronti di queste credenze, poi progressivamente addomesticate per poterle usare a proprio vantaggio sui fedeli, Lecouteux, Marcq 1990, pp. 7-13. Si rimanda anche a Schmitt 1988b, p. 473; Lauwers 1996.

${ }^{45}$ Schmitt 1994, pp. 79, 90-92.

${ }^{46}$ Già questo ci porta a considerarlo un fantasma vero e proprio, sensibilmente diverso dalla tipologia del revenant per cui il ritorno alla vita si configurava come un'opportunità del tutto temporanea e non irreversibile. Sulle tipologie, $c f$. Lecouteux 2009; 1998, pp. 195-222; Dias 2015 , p. 15.

${ }^{47}$ Sono quegli elementi che si organizzano in quello che la medievista Nancy Mandeville Caciola chiamava "a core of ideas", il quale ha a che fare "with the terms of existence for the dead (...) the belief that the dead live on in embodied form, in this world; that they form social groups, and are active chiefly at night in places familiar to them in life" (Caciola 2014, p. 328). Sulla costante della prossimità spirituale o anche solo spaziale tra vivo e morto, $c f$. anche Schmitt 1994, p. 97 (in riferimento proprio alle storie compilate dall'abate cluniacense) e Dias 2015, p. 14.

${ }^{48}$ A conferma di come l'elemento della relazione di prossimità (sociale o anche solo spaziale) tra il beneficiario dell'apparizione e lo spirito stesso sia uno dei capisaldi di lunga durata che rende possibile il verificarsi di questo tipo di fenomeni in un sistema di credenze popolari 
Il miraculum è quello rubricato De milite mortuo qui apparuit Humberto Beliocensi ('Il cavaliere morto apparso a Umberto di Beaujeau') che, come ci informa in apertura lo stesso Pietro, venne da lui registrato durante il periodo in cui si trovava in Spagna. Di seguito, si riproducono gli snodi fondamentali del testo latino ${ }^{49}$ :

dominum Guicardus hereditario jure suscipiens, eos quibus successerat patres, licet strenuos viros, saeculari potentia famaque excessit (...) dies vitae suae ex maxima parte vanitati et mundanae superbiae impendendo, multoque labore velut aranearum telas texendo, consumpsit. Tandem, diuturno morbo a Deo correptus ipsaque diuturnitate de vita diffidens, Cluniaci meo tempore monachus factus est. Ubi, corde humiliato, poenitentia et confessione Christiana, quantum datum fuerat, Deo satisfaciens, orationibus se sanctorum fratrum committens, paucis diebus advixit, atque ad ultimum peregrini itineris viatico, hoc est sacro Christi corpore suscepto, diem vitae ultimum clausit. Huic in paterna hereditate filius ejus Humbertus succedens, adolescentia et divitiis velut fortibus catenis mundo astrictus, spatiosas saeculi vias damnosa libertate aliquandiu effrenis incessit (...) Praelio dirempto, ad sua quisque vel fugiens vel persequens recessit. Necdum plene duo menses transierant, et ecce jam dictus miles, quem interfectum retuli, cuidam alteri militi de Ansa, qui Milo vocabatur, per silvam eidem castro contiguam sine socio equitanti, se obvium obtulit. Ad cujus subitum conspectum dum miles obstupuisset, et timore non modico turbatus, utrum fugere an subsistere sibi satius esset, meditaretur, mortuus qui apparuerat, prior in verba prorupit: Noli, ait, timere, neque effugere mediteris, quia non ut ego tibi noceam, sed ut tu mihi prodesse studeas, ad te venire permissum sum. Ad te enim prae omnibus veniendi licentiam accepi (...) Rogo igitur ut legationem meam Humberto de Belioco deferas (...) Nam, si missarum celebratione, eleemosynarum largitione, bonorum virorum apud Deum intercessione nos juvare studuerit, et celeriorem nobis salutem parabit, et seipsum debito quo nobis astringitur liberabit. Quod si tibi aut non crediderit, aut non obtemperaverit, necesse jam mihi erit per memetipsum eum adire, atque ei ut haec adimpleat instare. Scias autem multum jam mihi profuisse, factam nuper Lugduni in synodali conventu publicam absolutionem,

adottate precocemente dal Cristianesimo, si ricorra a quella che si conosce come una delle "richest and most fascinating collections of early ghost stories in any medieval text": il Chronicon del vescovo Thietmar di Merseburg (1013-1018). Nel celebre racconto del sabba di Walsleben, in occasione di una messa di morti riunitisi presso la chiesa in rovina della città (precedentemente passata a fil di spada assieme ai suoi abitanti), il prete protagonista della scoperta avanza tra le schiere mortuorum, incuriosito dall'evento. Come riporta Thietmar, l'unica entità che si rivolge al vivo è una donna morta che il prete conosceva bene e che -dettaglio ulteriormente importante- era deceduta da pochissimo tempo; $c f$. Caciola 2014, pp. 312-313. Su questi casi, $c f$. anche Berlioz, Ribaucourt 1993, pp. 25-26.

${ }^{49}$ Per il testo completo, si rimanda a Migne 1890, pp. 900-903. 
unde talium et consimilium tan ardenter deprecor subventionem.

Dixit haec, et statim a conspectu ejus evanuit.

Questo dunque il riassunto: un certo Goffredo d'Iden, cavaliere deceduto in una recente battaglia, appare ad un collega, Milone d'Anse. La sua iniziativa non si rivela in realtà del tutto personale: viene inviato sulla terra da quello che in vita era stato il suo signore, Guiscardo di Beaujeau. Lo spettro di Goffredo riceve infatti l'incarico di avanzare una richiesta a Milone, ossia quella di recarsi al cospetto del figlio ed erede di Guiscardo (Umberto) per informarlo del prodigio e chiedergli di intercedere per lui e per il padre con elemosine, messe e preghiere. A seguito del rifiuto dell'incarico da parte di Umberto, Goffredo decide di superare da solo l'impasse comparendo lui stesso davanti al figlio del suo signore per convincerlo ad operare per la loro salvezza.

Si può notare fin da subito come la trama della CSM 72 richiami sorprendentemente quella della prima parte di questo exemplum petrino dove il fantasma interviene nel mondo dei vivi su mandato di un altro defunto (che non appare) per facilitargli l'uscita dal Purgatorio; e lo fa attraverso la richiesta di appositi suffragi ed opere pie direttamente al cosiddetto nuncius (nella CSM tale funzione è rivestita dal padre del peccatore). Ma nella letteratura esemplare che contempla questo modello complesso, spesso pure il morto afferma di doversi salvare, alla pari di chi gli incarica l'apparizione. La sua si potrebbe dunque configurare come una specie di prova straordinaria di discesa sulla terra per potersi guadagnare il Regno dei Cieli.

Di casi come questi, in cui il fantasma veste i panni di una specie di nuncius, lasciando operare effettivamente l'individuo vivente al quale appare per la prima volta, se ne registrano pochi nella letteratura miracolistica medievale e quasi tutti confinati all'opera di Pietro il Venerabile. Questi aspetti si ritrovano compatti ancora nella tipologia di racconti che -grazie soprattutto all'impulso che ne danno gli ordini cistercensi a partire dal XII sec.- possono rientrare nella categoria delle cosiddette revelationes mortuorum per fissare progressivamente il concetto del Purgatorio tra i fedeli ${ }^{50}$.

Tornando ora al nostro problema, si può dunque ipotizzare che, alla pari del gambler della CSM 72 -che forse, in una più articolata versione del $m i$ -

\footnotetext{
${ }^{50} \mathrm{Su}$ questo aspetto e per un' analisi più approfondita di questo modello narrativo, si veda Schmitt 1988a, pp. 191-192. Sul Purgatorio e sulle dinamiche religiose preposte alla sua creazione, $c f$. Le Goff 1981. Si ricordi anche quanto ricordato da Fernando Martínez Gil in merito al Lucidario, uno dei testi castigliani dove si tratta da vicino l'argomento. Esso indica infatti, riflettendo pienamente la cultura del tempo, le motivazioni che portano anche le anime del Purgatorio ad apparire tra i vivi (eventualità tra l'altro ricordata anche da San Tommaso D'Aquino poco tempo dopo): "nos paresçen por demandarnos ayuda para sallir de aquella pena en que están, e este demandar fazen ellas amostrando el mal que han (...) " (Martínez 1996, p. 124).
} 
raculum, anteriore a quella della fonte dello Speculum, avrebbe potuto anch'egli incaricare il fantasma del vicino di comunicare col padre-, anche il suo vicino che appare nel bel mezzo del sentiero per ammonirlo (ma anche per aiutarlo) lo faccia per guadagnarsi il suo pezzo di Cielo ${ }^{51}$. Questo almeno, al di là della misericordia divina, renderebbe a tutti gli effetti comprensibile la sua apparizione ed offrirebbe una risposta dotata di senso al perché la Vergine non fosse stata lei stessa a manifestarsi per comunicare la posizione del ragazzo ucciso.

Per legittimare la sua apparizione nell'ottica delle sue finalità, non basterebbe dunque solo riconoscere che un ampio ventaglio di motivi, già codificati in modo sistematico nel Motif Index di Stith Thompson, dia notizia della possibilità dell'apparizione di morti spinti da buone intenzioni nel corso della cultura precristiana ${ }^{52}$ : occorre leggere tutto questo attraverso quella dottrina della redenzione sempre presente nell'opera mariana alfonsina per scorgere il perché di quelle stesse buone intenzioni, le ragioni del messaggio e la logica del 'premio celeste' che ne soggiaceva, forse anche per l'ambasciatore ancora errante tra i vivi ${ }^{53}$.

\section{OSSERVAZIONI E CONCLUSIONI}

Alla luce di quanto detto sia in merito ai debiti letterari che culturali del motivo dell'apparizione spettrale all'interno della CSM 72, si possono confermare le intuizioni della critica precedente in merito quantomeno alla non identità del fantasma col peccatore giustiziato per un peccato di blasfemia. Ma lo studio delle fonti dirette del miracolo versificato in galego-portoghese da Alfonso X e i suoi collaboratori ha permesso di fornire qualche ulteriore ipotesi sulla figura fantasmagorica che appare davanti al padre del protagonista per comunicargli la posizione del cadavere di quest'ultimo, nonché sulle presunte finalità che giustificherebbero tale manifestazione.

\footnotetext{
${ }^{51}$ Quello dell'aparecido in cerca di suffragio è inoltre uno dei modelli più diffusi nella letteratura esemplare, che rivaleggia solo con quel tipo di apparizioni aventi finalità di terrorizzare il malcapitato di turno; $c f$. Dias 2015, p. 16.

${ }^{52} \mathrm{Si}$ tratta di quel gruppo di motivi che, indicizzati nel Motif Index di Stith Thompson, vanno dal E300 fino al E399; $c f$. Thompson 1955-1958.

${ }^{53}$ Sulla legittimità di questa dinamica in un contesto culturale e letterario più ampio, si tenga ancora presente quanto osservato puntualmente in Caciola 2014, p. 330: "Thus it is tempting to suggest that the alms of the dead, viewed from within a Christian framework, are helping to support intercessions on their own behalf". D'altronde, come ricordato più volte da Jean-Claude Schmitt, nelle storie di spettri occorre sempre ricercare una costante comune che ha a che fare con tutta una serie di rituali della morte che, soprattutto a partire dai secoli centrali del Medievo in avanti, vengono recuperati dalla cultura ufficiale da credenze di lunga durata per trovare finalmente pieno riconoscimento durante l'epoca feudale; $c f$. Schmitt 1988a, p. 182.
} 
Analizzando infatti la prosa latina dello Speculum Historiale -sicuro modello per il Rei Sabio- si è potuto confermare pienamente il senso dell'aggettivo natural presente nella CSM 72, che indica appunto, come reso più esplicito dalla fonte seguita, una situazione di familiarità tra il vivo e il defunto. Tale identificazione permette di svelare inoltre alla base della riproposizione alfonsina del motivo tutta una serie di credenze e convinzioni diffuse durante il Medioevo che non solo ne spiegano la possibilità di apparire, rendendo così l'inserto narrativo alfonsino pienamente legittimo per l'epoca (come, ad esempio, lo stesso rapporto di vicinanza e la morte avvenuta da poco tempo), ma anche una probabile finalità a monte di tutta una tradizione miracolistica.

Non solo, infatti, il tutto deve essere letto come un aiuto gratuito che il fantasma (probabilmente su mandato divino, o comunque della stessa Vergine Maria mossa a pietà per le sorti del giovane blasfemo) offre al padre del giocatore affinché, una volta recuperato il suo corpo, possa seppellirlo e fare tutto il possibile per darsi pace nel provare a liberarlo dalle pene infernali. Alla luce del modello narrativo petrino che si è portato a confronto, il racconto potrebbe infatti anche celare un significato originario che farebbe del messaggero fantasma un individuo bisognoso di espiare le proprie colpe con un ultimo atto di carità cristiana ${ }^{54}$, nell'ottica di uno scambio di favori che si deve instaurare tra un vivo e un morto ${ }^{55}$. Forse è proprio questo $l$ 'aspetto nascosto che Ariel Guiance aveva intuito notando, nel Códice Rico, la raffigurazione dello spettro come totalmente incorporea; un'incorporeità che era incompletezza di redenzione e che comunicava l'urgenza per l'anima in pena di compiere un ultimo atto di devozione per liberarsi della pesantezza del purgatorio terreno in cui era confinato ${ }^{56}$.

\footnotetext{
${ }^{54}$ In molti racconti di apparizioni, anche più tardi della CSM 72, qualora un vincolo di qualsiasi tipo tra quelli tradizionalmente riconosciuti alla base delle manifestazioni dall'oltretomba (religioso, economico, vassallatico, ecc.) sia del tutto assente, nel testo appare quasi sempre specificato. Sebbene la CSM ricerchi la brevitas, omettendo dettagli presenti nelle fonti portate a collazione, in questa non accade, suggerendo la possibilità che effettivamente un legame anche minimo tra il fantasma e il padre del peccatore fosse cosa scontata e riconosciuta, causando a sua volta la possibilità -fatto che si dava quasi esclusivamente in presenza dei suddetti legami- che il modello originario contemplasse anche un favore del vivo per alleviare le pene purgatoriali del morto. Su questo, è illustrativo lo studio di Mahiques 2005, p. 25, che, indagando le fonti del celebre Somni di Berant Metge, offre una interessante casistica al riguardo. 1987.

${ }_{5}^{5}$ Sui favori tra vivi e i morti, tema ricorrente in questo tipo di letteratura, $c f$. Dinzelbacher

${ }^{56}$ Cf. Guiance 1998, p. 408.
} 


\section{BIBLIOGRAFIA CITATA}

Alcalá, Angel (1996), Doctrina teológica y leyendas pías en las Cantigas de Santa María de Alfonso X el Sabio, "Anuario medieval” 8, pp. 7-42.

Barre, Henri (1966), L'énigme du Mariale Magnum, "Ephemerides mariologicae" 16 , pp. 265-288.

Berlioz, Jacques; Ribaucourt, Colette (1993), Mors est timenda. Mort, morts et mourants dans la prédication médiévale. L'exemple de l'Alphabet des récits d'Arnold de Liège (début XIV siècle), in A Réveiller les morts. La mort a quotidien dans l'Occident médiéval. Préface de Jean Delumeau, Lyon, Presses universitaires de Lyon - Association des amis des bibliohèques de Lyon, pp. 17-30.

Brea, Mercedes (2002), Santiago y María: el milagro del peregrino engañado por el diablo, in Sub luce florentis calami. Homenaje a Manuel C. Díaz y Díaz, Santiago de Compostela, Universidade de Santiago de Compostela, pp. 591-607.

Brea, Mercedes (2005), Un gran milagro y sus versiones en las colecciones iberorrománicas de los Milagros de Santiago, in El Inglés como vocación. Homenaje al profesor Miguel Castelo Montero, A Coruña, Universidade da Coruña, pp. 93-100.

Brea, Mercedes (dir.) (2018-), Base de datos da Lírica Profana Galego-Portuguesa (MedDB) Versione 3.6, Santiago de Compostela, Centro Ramón Piñeiro para a Investigación en Humanidades, http://www. cirp.gal/meddb [consultazione: 18/03/2019].

Caciola, Nancy (2014), Revenants, Resurrection, and Burnt Sacrifice, "Preternature: Critical and Historical Studies on the Preternatural" 3/2, pp. 311-338.

Carpenter, Dwayne E. (1998), 'Alea jacta est': At the Gaming Table with Alfonso the Learned, "Journal of Medieval History" 24, pp. 333-345.

Cousteau, Nicolas; Du Pré, Galliot; De la Garde, Jean; Petit, Jean (eds.) (1531), Le premier volume de Vincent Miroir hystorial nouvellement imprime a Paris, Parigi, Galliot du Pré.

Davis, William R. (1974), Another Aspect of the Virgin Mary in the Cantigas de Santa Maria, "Revista de Estudios Hispánicos" 8, pp. 95-105.

Delcorno, Claudio (1989), I figli che saettano il padre, in "Exemplum” e letteratura. Tra Medioevo e Rinascimento, Bologna, il Mulino, pp. 163191.

Dias, Marta Miriam Ramos (2015), Os outros na Idade Média: fantasmas e "revenants", "Cultura, espaço \& memória" 6, pp. 11-21.

Dinzelbacher, Peter (1987), Le vie per l'Aldilà nelle credenze popolari e nella concezione erudita del Medioevo, "Quaderni medievali" 23, pp. 6-35. 
Domínguez, Cesar (1998), Vincent de Beauvais and Alfonso the Learned, "Notes and Queries" 45/2, pp. 172-173.

Faye Wilson, Evelyn (1946), The Stella Maris of John of Garland. Edited, Together with a Study of Certain Collections of Mary Legends Made in Northern France in the Twelfth and Thirteenth Centuries, Cambridge - Massachusetts, The Mediaeval Academy of America.

Fernández Fernández, Laura (2008-2009), Cantigas de Santa María: fortuna de sus manuscritos, "Alcanate. Revista de estudios Alfonsíes" 6, pp. 323-348.

Fernández Fernández, Laura (dir.) (2011), Las Cantigas de Santa María: Códice Rico, Ms. T-I-1, Real Biblioteca del Monasterio de San Lorenzo de El Escorial, vol. 1, Madrid, Patrimonio Nacional - Editorial Testimonio.

Ferreiro, Manuel (dir.) (2018-), Universo Cantigas. Edición crítica da poesía medieval galego-portuguesa, A Coruña, Universidade da Coruña, http://universocantigas.gal [consultazione: 18/03/2019].

Ferreiro, Manuel; Martínez Pereiro, Carlos Paulo; Tato Fontaiña, Laura (edd.) (2007), Normas de edición para a poesía trobadoresca galego-portuguesa medieval. Guidelines for the Edition of Medieval GalicianPortuguese Troubadour Poetry, A Coruña, Servizo de Publicacións da Universidade de Coruña.

Fidalgo, Elvira (2002a), As Cantigas de Santa Maria, Vigo, Xerais.

Fidalgo, Elvira (coord.) (2002b), As Cantigas de Loor de Santa María (edición e comentario), Santiago de Compostela, Centro Ramón Piñeiro para a Investigación en Humanidades.

Fidalgo, Elvira (2013), El Diablo en las Cantigas de Santa María, in Uno de los buenos del reino: homenaje al prof. Fernando D. Carmona, San Millán de la Cogolla, Cilengua, pp. 229-248.

Forsythe Dexter, Elise (1926), Sources of the Cantigas of Alfonso el Sabio, Madison, University of Wisconsin (tesi di dottorato inedita).

Fournès, Ghislaine (2015), La Vierge et l'héretique dans les miniatures du Códice Rico ('Cantigas de Santa María' d'Alphonse X de Castille, XIII ${ }^{e}$ siècle), "Les Cahiers de Framespa" 20, http://framespa.revues. org/3450 [consultazione: 21/01/2019].

França, Susani Silveira Lemos (1999), A intercessão mariana em prol da vida nas Cantigas de Santa Maria, in Para sempre em mim: Homenagem à Professora Ângela Vaz Leão, Belo Horizonte, Pontificia Universidade Católica de Minas Gerais, pp. 488-498.

Gallica = Bibliothèque nationale de France, http://gallica.bnf.fr [consultazione: $18 / 03 / 2019]$. 
Gripkey, Mary Vincentine (1952), Mary Legends in Italian Manuscripts in the Major Libraries of Italy, "Medieval Studies" 14, pp. 9-47.

Guiance, Ariel (1998), Los discursos sobre la muerte en la Castilla medieval (siglos VII-XV), Valladolid, Junta de Castilla y León - Consejería de Educación y Cultura.

Keller, John Esten (1988-1989), The Living Corpse: Miracle 67 of the CSM of Alfonso X, "Bulletin of the Cantigueiros de Santa Maria" 2, pp. 55-67.

Keller, John Esten (1991), El cadáver viviente: Milagro 67 de las Cantigas de Santa María de Alfonso el Sabio, in Homenaje a Alonso Zamora Vicente. III Literaturas medievales. Literatura española de los siglos XV-XVII, Madrid, Castalia, vol. 3-I, pp. 49-59.

Keller, John Esten; Kinkade, Richard P. (1984), Iconography in Medieval Spanish Literature, Kentucky, The University Press of Kentucky.

Lauwers, Michel (1996), La Mémoire des ancêtres, le souci des Morts: Morts, rites, et société au Moyen Âge, Parigi, Beauchesne.

Le Goff, Jacques (1981), La naissance du Purgatoire, Parigi, Gallimard.

Lecouteux, Claude (2009), Fantômes et Revenants au Moyen Âge. Postface de Régis Boyer, Paris, Éditions Imago.

Lecouteux, Claude (1998), Au-delà du merveilleux. Essai sur les mentalités du Moyen Âge, Parigi, Presses de l'Université de Paris-Sorbonne.

Lecouteux, Claude; Marcq, Philippe (1990), Les esprits et les morts. Textes traduits du latin, présentés et commentés, Parigi, Honoré Champion.

Mahiques Climent, Joan (2005), Lo somni de Bernat Metge i els tractats d'apareguts, "Llengua \& Literatura" 16, pp. 7-31.

Marchand, James W. (1998), Vincent de Beauvais, Gil de Zamora et le Mariale magnum, in Discours et savoirs: encyclopédies médiévales, Rennes, Presses Universitaires de Rennes, pp. 101-115.

Marroni, Giovanni (1968), Le poesie di Pedr' Amigo de Sevilha, "AIONAnnali dell'Istituto Orientale di Napoli. Sezione Romanza" 10, pp. 189-339.

Martínez Gil, Fernando (1996), La muerte vivida. Muerte y sociedad en Castilla durante la Baja Edad Media, Toledo, Diputación Provincial.

Mettmann, Walter (ed.) (1986), Alfonso X, el Sabio. Cantigas de Santa María. Cantigas de 1 a 100. I. Edición, introducción y notas, Madrid, Clásicos Castalia.

Migne, Jacques-Paul (ed.) (1890), Petri Venerabilis Abbati Cluniacensis Noni. Opera Omnia. Accedunt Wibaldi Abbatis Bonaevallis Epistolae et Opuscula, Parigi, Garnier Fratres Editores et J.-P Migne successores (Patrologia Latina; 189). 
Montoya Martínez, Jesús (1998-1999), La caballería blanca o caballería 'celeste' en la literatura hispánica, "La corónica” 27/2, pp. 7-19.

Moretti, Franco (2001), L'arte di far ridere sorridendo, in La ragione del sorriso e del riso nel Medioevo. Prefazione di Franco Porsia, Bari, Edipuglia, pp. 101-119.

Negri, Manuel (2017), Le Cantigas dei santi nello scriptorium del re Alfonso $X$ Il Saggio, Santiago de Compostela, Universidade de Santiago de Compostela (tesi di dottorato inedita).

Paredes Núñez, Juan (ed.) (2010), El cancionero profano de Alfonso X el Sabio: edición crítica, con introducción, notas y glosario, Santiago de Compostela, Universidade de Santiago de Compostela.

Parkinson, Stephen (dir.) (2005-), The Oxford Cantigas de Santa Maria Database, http://csm.mml.ox.ac.uk/?p=database [consultato: 18/03/ 2019].

Parkinson, Stephen (2010), Questões de estrutura estrófica nas Cantigas de Santa María: estruturas múltiplas, assimetrias e continuações inconsistentes, in Estudos de edición crítica e lírica galego portuguesa, Santiago de Compostela, Servizo de Publicacións e Intercambio Científico, pp. 315-336.

Parkinson, Stephen (2011), Alfonso X, miracle collector, in Las Cantigas de Santa María: Códice Rico, Ms. T-I-1, Real Biblioteca del Monasterio de San Lorenzo de El Escorial, Madrid, Patrimonio Nacional - Editorial Testimonio, pp. 79-105.

Parkinson, Stephen (ed.) (2015), Alfonso X the Learned. Cantigas de Santa Maria. An anthology, Londra, MHRA.

Parkinson, Stephen; Jackson, Deirdre (2006), Collection, Composition and Compilation in the Cantigas de Santa Maria, "Portuguese Studies" 22/2, pp. 159-172.

Paulmier-Foucart, Monique (2004), Vincent de Beauvais et le Grand Miroir $d u$ Monde, avec la collaboration de Marie-Christine Duchenne, Turnhout, Brepols (Témoins de Notre Histoire; 10).

Poncelet, Albert (1902), Index miraculorum B. V. Mariae quae saec. VI-XV latine conscripta sunt, "Analecta Bollandiana" 21, pp. 242-360.

Rubio García, Luis (1985), En torno a la biblioteca de Alfonso X el Sabio, in La lengua y la literatura en tiempos de Alfonso X: Actas del Congreso Internacional (Murcia, 5-10 marzo 1984), Murcia, Universidad de Murcia, pp. 531-551.

Schaffer, Marta (1999), Los códices de las Cantigas de Santa María: su problemática, in El Escriptorium alfonsí: de los Libros de Astrología a las "Cantigas de Santa María", Madrid, Editorial Complutense, pp. 126-148. 
Schmitt, Jean-Claude (1982), Les revenants dans la société féodale, in Le Temps de la réflexion ( $\left.n^{\circ} 3\right)$, Parigi, Gallimard, pp. 285-306.

Schmitt, Jean-Claude (1987), Le spectre de Samuel et la sorcière d'En Dor. Avatars historiques d'un récit biblique: I Rois 28, "Études rurales" 105-106, pp. 37-64.

Schmitt, Jean-Claude (1988a), Gli spettri nella società feudale, in Religione, folklore e società nell'Occidente medievale, Bari, Laterza, pp. 182205.

Schmitt, Jean-Claude (1988b), Les 'superstitions', in Histoire de la France religieuse, Tome 1: Des dieux de la Gaule à la papauté d'Avignon (des origines au XIV siècle), Parigi, Éditions du Seuil, pp. 417-551.

Schmitt, Jean-Claude (1994), Les Revenants: les vivants et les morts dans la société médiévale, Parigi, Gallimard.

Schmitt, Jean-Claude (2013), Medioevo superstizioso. Traduzione di Maria Garin, Bari, Laterza.

SourcEncyMe $=$ Sources des encyclopédies médiévales $(\mathrm{CNRS}$, Institute de recherche et d'histoire des textes), http://sourcencyme.ihrt.cnrs.fr [consultazione: 26/07/2019].

Taylor, Barry (2015), Alfonso X y Vicente de Beauvais, in Literatura y ficción: 'estorias', aventuras y poesía en la Edad Media, València, Publicacions Universitat de València, vol. 2, pp. 447-458.

Thesaurus Exemplorum Medii Aevi = ThEMA (CNRS, Groupe d'Anthropologie Historique de l'Occident Mediéval), http://gahom.huma-num.fr/ thema [consultazione: 26/07/2019].

Thompson, Stith (1955-1958), Motif-index of Folk Litterature: A Classification of Narrative Elements in Folktales Ballads, Myths, Fables, Mediaeval Romances, Exempla, Fabliaux, Jestbook and Local Lagends. Revised and Enlarged Edition, 6 voll., Bloomington, Indiana University Press.

Tubach, Frederic C. (1981), Index exemplorum: a handbook of medieval religious tales, Helsinki, Academia Scientiarum Fennica.

Vauchez, André (1981), La saintété en Occident aux derniers siècles du Moyen Âge, Roma, École française de Rome.

Vauchez, André (2009), Fenomenologia dell'evento miracoloso: le sue strutture e la sua estensione, in La santità nel Medioevo, Bologna, il Mulino, pp. 447-485.

Voorbij, Johannes B. (1990), La version Klosterneuburg et la version Douai du "Speculum historiale": manifestations de l'évolution du texte, in Paulmier-Foucart, Monique; Lusignan, Serge; Nadeau, Alain (edd.), Vincent de Beauvais: Intentions et récepcions d'une aeuvre encyclopédique au Moyen Âge. Actes du XIV Colloque de l'Institut 
d'études médiévales, organisé conjointement par l'Atelier Vincent de Beauvais (A.R.Te.M., Université de Nancy II) et l'Institut d'études médiévales (Université de Montréal) 27-30 avril 1988, Ville SaintLaurent - Parigi, Les Éditions Bellarmin, 1990, pp. 111-140.

Voorbij, Johannes B. (1997), Les mises à jour de la matière dominicaine dans le Speculum historiale, in Lusignan, Serge; Paulmier-Foucart, Monique (edd.), Lector et compilator: Vincent de Beauvais, frère prêcheur un intellectuel et son milieu au XIII siècle, Grane, Créaphis, pp. 153168.

Ward, Harry Leigh Douglas (1893), Catalogue of romances in the Department of manuscripts in the British Museum, vol. 2, Londra, Oxford University Press.

Fecha de recepción del artículo: marzo 2019

Fecha de aceptación y versión final: octubre 2019 